\title{
CONTINUOUS BOUNDARY VALUES OF ANALYTIC FUNCTIONS OF SEVERAL COMPLEX VARIABLES
}

\author{
BARNET M. WEINSTOCK ${ }^{1}$
}

1. Let $D$ be a relatively compact domain in $C^{n}, n>1$, with smooth boundary $\Gamma$. Let $A(\Gamma)$ be the algebra of continuous, complex-valued functions on $\Gamma$ which have analytic extensions to $D$. In this note we derive the following characterization of $A(\Gamma)$ :

THEOREM. A continuous function $f$ on $\Gamma$ belongs to $A(\Gamma)$ if and only if

$$
\int_{\mathbf{r}} f \omega=0
$$

for each $(n, n-1)$ form $\omega$ which is $C^{\infty}$ in $D \cup \Gamma$ and satisfies $\bar{\partial} \omega=0$ in $D$.

Our method is elementary, and was suggested by the proofs of the Hartogs' Extension Theorem given by Bochner [1] and Martinelli [6]. We remark that the differential forms $\omega$ appearing in the theorem are a natural generalization to several variables of the "analytic differentials" which play the corresponding role in the well-known one-variable theorem. By appealing to the regularity theorem of Kohn [4] for the $\bar{\partial}$-Neumann problem we obtain as a corollary that if the Levi form on $\Gamma$ has at least one positive eigenvalue at each point, then $f \in A(\Gamma)$ if and only if $f$ is a weak solution of the tangential Cauchy-Riemann equations. (With this hypothesis on $\Gamma$, it follows that if $D$ is connected, then so is $\Gamma$.)

This theorem was obtained by Fichera [2] under the additional hypotheses that $\Gamma$ is connected and $f$ is the boundary value of a function with finite Dirichlet integral. His proof is based on an approximation theorem for harmonic functions in the Dirichlet norm. Kohn and Rossi [5] have shown that the theorem holds for $C^{\infty}$ functions when $D$ is a finite domain on a complex manifold and the Levi form satisfies the above condition on $\Gamma$. They also obtain extension theorems for $(p, q)$ forms.

2. We assume a basic familiarity with the algebra of complex differential forms. If $S$ is an open set in $C^{n}$ we denote by $E^{p, q}(S)$ the space of $C^{\infty}$ forms of bidegree $(p, q)$ on $S$. The usual exterior differential operator, $d$, splits as the direct sum $\partial+\bar{\partial}$, where $\partial: E^{p, q} \rightarrow E^{p+1, q}$ differentiates with respect to $z_{1}, \cdots, z_{n}$, and $\bar{\partial}: E^{p, q} \rightarrow E^{p, q+1}$ differ-

Received by the editors June 12, 1968.

1 This work was partially supported by NSF Grant GP-7653. 
entiates with respect to $\bar{z}_{1}, \cdots, \bar{z}_{n}$. We recall that the Hodge star operator maps $E^{p, q}$ isomorphically onto $E^{n-q, n-p}$. For 1 -forms we have the formulas

$$
{ }^{*} d z_{k}=-2(i / 2)^{n} d z_{k} \wedge \omega_{k}, \quad{ }^{*} d \bar{z}_{k}=2(i / 2)^{n} d \bar{z}_{k} \wedge \omega_{k}
$$

where $\omega_{k}=\prod_{i \neq k} d z_{i} \wedge d \bar{z}_{i}$. If $g \in C^{\infty}(S)$ it follows that

$$
* d g-2 * \partial g=2(i / 2)^{n} d g \wedge \sum_{k=1}^{n} \omega_{k} .
$$

In particular if $S$ is a neighborhood of the compact $(2 n-1)$-manifold $\Gamma$, and $g=0$ on $\Gamma$, then the $(2 n-1)$-forms $* d g$ and $2^{*} \partial g$ define the same measure on $\Gamma$.

Every continuous function $f$ on $\Gamma$ has a harmonic extension to $D$ given by

$$
f(\zeta)=-(n-2) ! \pi^{-n} \int_{\Gamma} f(z)^{*} d G(z, \zeta)
$$

where $G(z, \zeta)$ is the Green's function for $D$. Since for fixed $\zeta \in D$ we have $G(z, \zeta)=0$, and also that $H(z, \zeta)=G(z, \zeta)-|z-\zeta|^{2-2 n}$ is harmonic throughout $D$, we can write

$$
\begin{aligned}
\frac{1}{2} f(\zeta)= & -(n-2) ! \pi^{-n} \int_{\Gamma} f(z)\left(* \partial|z-\zeta|^{2-2 n}+* \partial H(z, \zeta)\right) \\
= & (-1)^{n}(n-1) !(2 \pi i)^{-n} \int_{\Gamma} f(z)|z-\zeta|^{-2 n} \sum_{i=1}^{n}\left(\bar{z}_{i}-\bar{\zeta}_{i}\right) d z_{i} \wedge \omega_{i} \\
& -(n-2) ! \pi^{-n} \int_{\Gamma} f(z)^{*} \partial H(z, \zeta)
\end{aligned}
$$

where, for each $\zeta, \bar{\partial}^{*} \partial H(z, \zeta)=0$.

Let $\omega_{i j}=\prod_{k \neq i, j} d z_{k} \wedge d \bar{z}_{k}$ and

$$
\Omega_{j}(\zeta)=\sum_{i=1}^{n}|z-\zeta|^{-2 n}\left(\bar{z}_{i}-\xi_{i}\right) d z_{j} \wedge d z_{i} \wedge \omega_{i j} .
$$

Since $\sum_{i=1}^{n} \partial\left(\bar{z}_{i}-\bar{\zeta}_{i}\right)|z-\zeta|^{-2 n} / \partial \bar{z}_{i}=0$, a straightforward computation shows that for $z \in D-\{\zeta\}$,

$$
\bar{\partial} \Omega_{j}(\zeta)=-\sum_{i=1}^{n} \frac{\partial}{\partial \bar{z}_{j}}\left(\bar{z}_{i}-\xi_{i}\right)|z-\zeta|^{-2 n} d z_{i} \wedge \omega_{i}
$$

For each $\zeta \in D$ we denote by $\Omega_{j}^{\prime}(\zeta)$ a form in $E^{n, n-2}(D)$ which agrees with $\Omega_{j}(\zeta)$ in a neighborhood of $\Gamma$. 
Proof of Theorem. Necessity follows from Stokes' Theorem, since if $f \in A(\Gamma)$ and $\bar{\partial} \omega=0$ in $D$, then

$$
\int_{\Gamma} f \omega=\int_{D} d(f \omega)=\int_{D} \bar{\partial}(f \omega)=\int_{D} \bar{\partial} f \wedge \omega+f \bar{\partial} \omega=0 .
$$

(Here we denote again by $f$ the analytic extension of $f$ to $D$.) To prove the converse, extend $f$ to a harmonic function in $D$. Since $f$ satisfies (1), the second integral in (2) vanishes, hence the harmonic extension is given by the Bochner-Martinelli formula, which is the first integral in (2). But then, for each $j$, we have

$$
\frac{1}{2} \frac{\partial f}{\partial \bar{\zeta}_{j}}(\zeta)=(-1)^{n}(n-1) !(2 \pi i)^{-n} \int_{\mathrm{r}} f(z) \bar{\partial} \Omega_{j}^{\prime}(\zeta) .
$$

Since $\bar{\partial} \Omega_{j}^{\prime}(\zeta)$ is a smooth, $\bar{\partial}$-closed $(n, n-1)$-form on $D \cup \Gamma$ for each $\zeta$, the integral vanishes by (1), hence $f$ is analytic. Q.E.D.

3. Definition. Let $g$ be a $C^{\infty}$ function defined in a neighborhood $N$ of $\Gamma$ such that:

(i) $\Gamma=\{g=0\}$.

(ii) $d g \neq 0$ on $\Gamma$.

(iii) $D \cap N=\{g<0\}$.

We say that $\Gamma$ is 1-strongly-pseudoconvex if at each point of $\Gamma$ the hermitian form $\sum \partial^{2} g / \partial z_{i} \partial \bar{z}_{j} a_{i} \bar{a}_{j}$ on the subspace defined by $\sum a_{j} \partial g_{l} / \partial z_{j}$ $=0$ has at least one positive eigenvalue.

If $f \in C^{\infty}(\Gamma)$, let $f^{\prime}$ denote an arbitrary $C^{\infty}$ extension of $f$ to a neighborhood of $\Gamma$. If the tangential component (in terms of a "boundary coordinate system") of $\bar{\partial} f^{\prime}$ vanishes on $\Gamma$ we say that $f$ is a solution of the tangential Cauchy-Riemann (C-R) equations. (See [5].) Equivalently, $f$ is a solution of the tangential C-R equations if $X\left(f^{\prime}\right)=0$ on $\Gamma$ for all complex vector fields $X$ of the form $\sum \phi_{j} \partial / \partial \bar{z}_{j}$ which are tangent to $\Gamma$.

Let $f$ be a solution of the tangential C-R equations and $\mu$ be a smooth $(n, n-2)$ - form in a neighborhood of $\Gamma$. Then

$$
\int_{\Gamma} f \bar{\partial} \mu=\int_{\Gamma} \bar{\partial} f^{\prime} \wedge \mu+f \bar{\partial} \mu=\int_{\Gamma} \bar{\partial}\left(f^{\prime} \mu\right)=0 .
$$

If $f$ is a continuous function on $\Gamma$ we say that $f$ is a weak solution of the tangential Cauchy-Riemann equations if the first integral above vanishes for all such $\mu$.

CoROllary. If $\Gamma$ is 1-strongly-pseudoconvex, then $f \in A(\Gamma)$ if and only if $f$ is a weak solution of the tangential $C-R$ equations. 
Proof. With this assumption on $\Gamma$ it follows that $H^{n-1}\left(D, \Omega^{n}\right)=0$ where $\Omega^{n}$ is the sheaf of germs of holomorphic $n$-forms on $D$. (See [3].) But by the Kohn regularity theorem [4]

$$
H^{n-1}\left(D, \Omega^{n}\right) \approx \frac{\left\{\omega \in E^{n, n-1}(D \cup \Gamma): \bar{\partial} \omega=0\right\}}{\left\{\partial \mu: \mu \in E^{n, n-2}(D \cup \Gamma)\right\}} .
$$

Thus for each $\omega$ in the hypothesis of the theorem there is an $(n, n-2)$ form $\mu$ which is $C^{\infty}$ in $D \cup \Gamma$ such that $\omega=\bar{\partial} \mu$.

We remark that if the domain $D$ in $C^{n}$ is connected and $\Gamma$ is 1-strongly-pseudoconvex, then $\Gamma$ is connected (cf. [5, Corollary 7.3]).

4. It is reasonable to conjecture that the theorem remains true for relatively compact domains on other complex manifolds, e.g., on Stein manifolds. For domains $D$ on Kähler manifolds (see [7] for definition) and $C^{\infty}$ boundary values $f$ there is the following simple proof:

We can extend $f$ to a harmonic function in $D$, i.e., a function satisfying $d^{*} d f=0$. Because of the Kähler metric this implies that $f$ also satisfies $\bar{\partial} * \partial \bar{f}=0$. (See [7].) Hence $\int_{\Gamma} f^{*} \partial \bar{f}=0$. But

and

$$
\int_{\Gamma} f^{*} \partial \bar{f}=\int_{D} \bar{\partial} f \wedge * \overline{\bar{\partial} f}
$$

$$
\|\mu\|=\left(\int_{D} \mu \wedge^{*} \bar{\mu}\right)^{1 / 2} \quad \mu \in E^{p, q}(D \cup \Gamma)
$$

is a norm on $E^{p, q}(D \cup \Gamma)$. Hence $\bar{\partial} f=0$ on $D$, i.e., $f$ is analytic.

\section{REFERENCES}

1. S. Bochner, Analytic and meromorphic continuation by means of Green's formula, Ann. of Math. 44 (1943), 652-673.

2. G. Fichera, Caratterizzazione della traccia, sulla frontiera di un campo, di una funzione analitica di piu variabili complesse, Atti Accad. Naz. Lincei Rend. Cl. Sci. Fis. Mat. Natur. (8) 22 (1957), 706-715.

3. L. Hörmander, $L^{2}$ estimates and existence theorems for the $\bar{\partial}$ operator, Acta Math. 113 (1965), 89-152.

4. J. J. Kohn, Harmonic integrals on strongly pseudoconvex manifolds. I, Ann. of Math. 78 (1963), 112-148.

5. J. J. Kohn and H. Rossi, On the extension of holomorphic functions from the boundary of a complex manifold, Ann. of Math. 81 (1965), 451-473.

6. E. Martinelli, Sopra una dimonstrasione di R. Fueter per un teorema di Hartogs, Comment. Math. Helv. 15 (1942), 340-349.

7. A. Weil, Variêtés Kähleriennes, Actualitês Sci. Indust., No. 1267, Hermann, Paris, 1958.

BROWN UNIVERSITY 